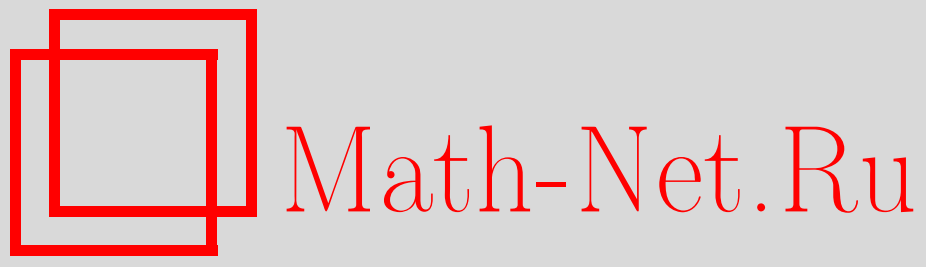

А. Н. Колмогоров, Отзыв о работах И. М. Гельфанда по унитарным представлениям непрерывных групп, Функи. анализ и его прил., 2003, том 37, выпуск 4, 57

DOI: https://doi.org/10.4213/faa3008

Использование Общероссийского математического портала MathNet.Ru подразумевает, что вы прочитали и согласны с пользовательским соглашением

http://www.mathnet.ru/rus/agreement

Параметры загрузки:

IP : 54.197 .130 .99

26 апреля 2023 г., 12:45:15

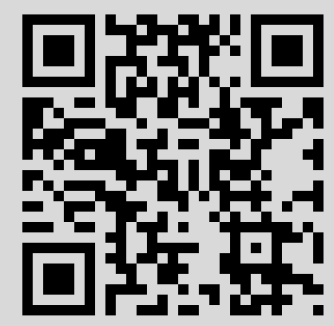


исследований. Их теория является естественной современной формой гармонического анализа функций и спектрального анализа детерминированных или случайных колебательных процессов. В недавних работах Дирака большое значение приобрели унитарные представления группы Лоренца.

Основой всей будущей теории унитарных групп и унитарных представлений должна явиться теорема Гельфанда и Райкова о существовании достаточной системы непрерывных унитарных представлений. После получения этой теоремы естественно возник вопрос о нахождении всех неприводимых представлений особенно важных групп и составлении любого представления из этих неприводимых. В этом направлении в качестве первого случая (представляющего существенные трудности) И. М. Гельфанд и М. А. Наймарк разобрали представления группы Лоренца. Найденная ими полная система неприводимых представлений дается в виде вполне обозримых формул. Все представления, найденные Дираком, оказались приводимыми и вполне обозримым образом составляются из неприводимых. Это исследование само по себе заслуживало бы самой высокой оценки. В работе [8] его результаты применены к выводу релятивистски инвариантных дифференциальных уравнений. В работе [4] получены менее исчерпывающие, но весьма глубокие результаты в направлении перечисления неприводимых представлений полупростых групп Ли.

Весь описанный круг исследований И. М. Гельфанда представляется мне безусловно самым крупным явлением в области функционального анализа в мировой математике последнего десятилетия и вполне заслуживает награждения Сталинской премией.

Приписано от руки: Так как работы [8-11] (в том числе работа [8], существенная для понимания значения и перспектив физических применений всего цикла работ И. М. Гельфанда) вышли в свет лишь в самом конце 1948 г., то представление всего цикла работ на премию в 1949 г. соответствует установленному для этого порядку.

A. Колмогоров 16 октября 1949 г.

\section{Отзыв о работах И. М. Гельфанда по унитарным представлениям непрерывных групп}

Вышедшая в 1950 г. монография И. М. Гельфанда и М. А. Наймарка [1] является завершением работы, начатой И. М. Гельфандом с рядом сотрудников в 1942 году. Общее число публикаций И. М. Гельфанда и его сотрудников на данную тему приближается к двадцати, однако основные результаты содержатся в представляемых на премию четырех работах, опубликованных с 1943 по 1950 год. Созданная в этих работах теория унитарных представлений групп является одним из самых замечательных достижений за последние десять лет в математической науке: она дает новый мощный метод для решения важных и трудных задач в весьма различных областях математики и математического естествознания. 
Для современной физики особенно большой интерес имеет применение общих методов Гельфанда к нахождению всех неприводимых унитарных представлений группы Лоренца (и получению из них всех остальных унитарных представлений этой группы). Эта задача полностью и вполне эффективно в смысле простого явного аналитического выражения неприводимых представлений решена в работе [3]. Применения к выводу общего вида релятивистски инвариантных дифференциальных уравнений даны в работе [4]. Эти вопросы после работы Дирака, опубликованной в 1945 году и содержавшей лишь случайные примеры бесконечномерных представлений группы Лоренца, которые все оказались разложимыми (см. введение к [3]), привлекают очень большое внимание также и иностранных исследователей, но первым по времени опубликования и самым полным решением задачи является решение, данное И. М. Гельфандом.

С общематематической стороны самыми существенными этапами работы следует признать

а) установление того, что для любой локально компактной группы существует полная система неприводимых унитарных представлений (этот результат изложен в статье [2] и послужил началом всего цикла исследований), и

б) выполненное вполне эффективными аналитическими средствами вычисление всех неприводимых представлений основных классических групп и доказательство того, что в случае этих групп все унитарные представления разлагаются на неприводимые, т. е. что обозрение всех унитарных представлений этих групп доставляется с полной ясностью перечислением их неприводимых представлений. Эта часть работы вместе с разработкой ее применений оказалась наиболее трудной в смысле аналитического аппарата и выполнена совместно И. М. Гельфандом и $М$. А. Наймарком. Ее результаты и составляют содержание фундаментальной монографии [1].

Явное выражение всех неприводимых представлений какой-либо группы приносит с собою решение целого ряда основных задач, связанных с этой группой. В частности, решается задача нахождения всех положительно определенных функций, инвариантных по отношению к преобразованию данной группы: дается их разложение на «элементарные» положительно определенные функции.

Для группы движений прямой такие элементарные положительно определенные функции исчерпываются функциями

$$
f(x)=e^{i \lambda x},
$$

и это составляет основу «гармонического анализа» функций одного переменного $x$. Группа вращений сферы порождает таким же образом сферические функции. В этих случаях неприводимые представления конечномерны, и указанные результаты были известны ранее работ И. М. Гельфанда. Но уже начиная с таких групп, как группа движений плоскости Лобачевского или группа преобразований подобия для полуплоскости, вопрос решается лишь при помощи бесконечномерных унитарных преобразований и теории И. М. Гельфанда. Построение такой обобщенной теории «сферических функций» составляет одну из существенных заслуг И. М. Гельфанда и М. А. Наймарка. Заметим в связи с этим, что явное выражение всех инвариантных положительно определенных функций решает 
в статистической механике непрерывных сред задачу нахождения явного вида всех инвариантных по отношению к данной группе корреляционных функций для связанных с точкой случайных величин.

Большой интерес имеют обобщения на классические непрерывные группы соотношения, которое в случае обычного гармонического анализа называется формулой Планшереля. Чтобы дать это соотношение во вполне эффективной форме (с явным выражением числового множителя в формуле (27.17)), например, для комплексной унимодулярной группы авторам работы [1] пришлось преодолеть большие трудности.

В виде одного из неожиданных совсем недавних применений методов теории И. М. Гельфанда мне хотелось бы еще указать на нахождение в еще не опубликованной работе И. М. Гельфанда и С. В. Фомина ${ }^{1)}$ спектров для динамических систем, получающихся из рассмотрения геодезических линий на поверхности постоянной отрицательной кривизны. Это первый случай полного определения непрерывного спектра классической динамической системы, явно заданной при помощи векторного поля на многообразии (в данном случае на трехмерном многообразии линейных элементов).

Следует отметить, что уже доказательство Хедлундом и Э. Хопфом того факта, что эти динамические системы имеют непрерывный спектр (неизвестной природы), рассматривалось как одно из самых замечательных событий в теории динамических систем.

Дело, впрочем, не в отдельных примерах подобного рода. Они лишь подчеркивают полную эффективность методов И. М. Гельфанда и их приспособленность к решению трудных конкретных задач. Вся же широта открывающихся здесь перспектив может быть оценена в полной мере лишь на основе понимания общепризнанного узлового положения классических непрерывных групп и их линейных представлений в математике. До работ И. М. Гельфанда мы владели должным образом лишь конечномерными (матричными) представлениями и представлениями, на них разлагающимися. Нахождение всех бесконечномерных унитарных представлений и доказательство того, что в основных классических случаях все унитарные представления разлагаются на неприводимые унитарные (конечномерные и бесконечномерные), является самым существенным расширением классической теории линейных представлений групп со времени создания ее основ.

1. Гельфанд И. М., Наймарк М. А. Унитарные представления классических групп. Труды МИАН им. В. А. Стеклова, 36, 1-288 (1950).

2. Гельфанд И. М., Райков Д. А. Неприводимые унитарные представления локально бикомпактных групп. Матем. сб., 13, 301-316 (1943).

3. Гельфанд И. М., Наймарк М. А. Унитарные представления группы Лоренца. Изв. АН СССР, сер. матем., 11, 411-504 (1946).

4. Гельфанд И. М., Яглом А. М. Общие релятивистски инвариантные уравнения и бесконечномерные представления группы Лоренца. ЖЭТФ, 18, 703-733 (1948).

1) Имеется в виду работа: Гельфанд И. М., Фомин С. В. Геодезические потоки на поверхностях постоянной отрицательной кривизны. УМН, 7, вып. 1, 118-137 (1952). 